\title{
Quantitative cell nuclear imaging by dual-view optical-resolution photoacoustic microscopy
}

Terence T. W. Wong, De Cai, Liren Zhu, Junhui Shi, Sung-Liang Chen, et al.

Terence T. W. Wong, De Cai, Liren Zhu, Junhui Shi, Sung-Liang Chen, Lihong V. Wang, "Quantitative cell nuclear imaging by dual-view opticalresolution photoacoustic microscopy," Proc. SPIE 10878, Photons Plus Ultrasound: Imaging and Sensing 2019, 108780R (27 February 2019); doi: $10.1117 / 12.2509219$

SPIE. Event: SPIE BiOS, 2019, San Francisco, California, United States 


\author{
Quantitative cell nuclear imaging by dual-view optical-resolution \\ photoacoustic microscopy \\ Terence T. W. Wong ${ }^{\mathrm{a}, \mathrm{b}}$, De Cai ${ }^{\mathrm{a}, \mathrm{c}}$, Liren Zhu ${ }^{\mathrm{a}}$, Junhui Shi ${ }^{\mathrm{a}}$, Sung-Liang Chen ${ }^{\mathrm{c}}$, and Lihong V. \\ Wang, ${ }^{\mathrm{a}, *}$ \\ ${ }^{a}$ Caltech Optical Imaging Laboratory, Andrew and Peggy Cherng Department of Medical \\ Engineering, Department of Electrical Engineering, California Institute of Technology, Pasadena, \\ CA 91125, USA; \\ ${ }^{b}$ Present address: Translational and Advanced Bioimaging Laboratory, Department of Chemical and \\ Biological Engineering, Hong Kong University of Science and Technology, Hong Kong, China; \\ ${ }^{\mathrm{c}}$ University of Michigan-Shanghai Jiao Tong University Joint Institute, Shanghai Jiao Tong \\ University, Shanghai 200240, China; \\ *Corresponding author email address: LVW@caltech.edu
}

\begin{abstract}
An estimated $~ 250,000$ new cases of both invasive and non-invasive breast cancer were diagnosed in US women almost every year. To reduce the local recurrence rate, the breast conserving surgery (BCS) is widely used as the initial therapy, which is to excise the tumor with a rim of normal surrounding tissue such that no cancer cells remain at the cut margin, Patients with positive margin commonly require a second surgical procedure to obtain clear margins. To this end, optical-resolution photoacoustic microscopy (OR-PAM) with ultraviolet (UV) laser illumination (OR-UV-PAM) has been developed for providing label-free, high-resolution, and histology like imaging of fixed, unprocessed breast tissue. To further improve the performance of OR-UV-PAM, here, we introduce dual-view UV-PAM (DV-UV-PAM) to significantly improve the axial resolution, achieving three-dimensional (3D) resolution isotropy. We first use $0.5 \mu \mathrm{m}$ polystyrene beads and carbon fibers to validate the resolution isotropy improvement. Imaging of mouse brain slices further demonstrates the improved resolution isotropy, revealing the 3D structure of cell nuclei in detail, which facilitates quantitative cell nuclear analysis.
\end{abstract}

Keywords: Photoacoustic microscopy, photoacoustic tomography, cell nuclear imaging, label-free imaging, quantitative imaging

\title{
1. INTRODUCTION
}

Optical-resolution photoacoustic microscopy (OR-PAM) has found broad applications in imaging biological objects ranging from organelles to organs [1]. The axial resolution in OR-PAM, which is determined by the bandwidth of an ultrasonic transducer matching the targeted tissue penetration, is typically limited to tens of micrometers. The anisotropic resolutions hindered OR-PAM from revealing accurate three-dimensional (3D) structures of biological tissue. OR-PAM with ultraviolet (UV) laser illumination has recently been exploited to image human breast and mouse brain tissues with specific cell nuclear contrast for cancer diagnosis and intraoperative margin assessment as well as for basic research in neuroscience $[2,3]$. The resolution anisotropy in OR-PAM with UV laser illumination is particularly severe due to the finer optical lateral resolution enabled by the shorter laser wavelength, especially at a high optical numerical aperture (NA) (e.g., $0.33 \mu \mathrm{m}$ lateral resolution versus $48 \mu \mathrm{m}$ axial resolution [2]).

In recent years, different approaches have been developed to enhance the axial resolution, thus improving the resolution isotropy of OR-PAM. Wide bandwidth ultrasonic transducers [4] and optical ultrasonic detectors [5] have been utilized for high-frequency ultrasonic detection to refine axial resolution. However, the severe attenuation of high-frequency acoustic waves in biological tissue limits the imaging depth and working distance. Moreover, optical sectioning has been achieved in OR-PAM by nonlinear effects such as Grüneisen relaxation [6, 7]. Yet, the imaging speed is reduced owing to the need for additional depth scanning.

Photons Plus Ultrasound: Imaging and Sensing 2019, edited by Alexander A. Oraevsky, Lihong V. Wang, Proc. of SPIE Vol. 10878, 108780R - (C) 2019 SPIE · CCC code: 1605-7422/19/\$18 - doi: 10.1117/12.2509219 


\section{METHODOLOGY}

\section{Dual-view optical-resolution photoacoustic microscopy (DV-OR-PAM)}

Here, we report dual-view OR-PAM (DV-OR-PAM) with UV laser illumination, developed to improve resolution isotropy. Instead of rotating the sample, linear scanning is employed in DV-OR-PAM, which facilitates the imaging of sliced biological samples. Only two views are acquired. The schematic of DV-OR-PAM is illustrated in Fig. 1. The 5mm-diameter UV laser beam (Wedge-HF-266, 266-nm wavelength, Bright Solutions SRL) is split into two orthogonal beams, named as the left and right view laser beams hereafter. The two beams are then focused onto the sample from below, each by an aspheric lens with an NA of 0.29 (AFL25-40-S-U, asphericon GmbH). The ultrasonic waves generated inside the sample through the photoacoustic effect are detected by an ultrasonic transducer (V358-SU, 50$\mathrm{MHz}$ central frequency, $88 \%$ bandwidth (one-way), Olympus NDT, Inc.) with a homemade concave lens at the front to provide acoustic focusing. After electrical amplification (56 dB, two ZFL-500LN+, Mini-Circuits, Inc.), the signal is digitized by a data acquisition card (ATS9350, Alazar Technologies, Inc.) at a sampling rate of $500 \mathrm{MS} / \mathrm{s}$ and sent to a computer for afterward processing. Each A-line signal is converted to a depth-resolved profile by taking the envelope. The water tank with the sample is raster scanned with a step size of $0.3125 \mu \mathrm{m}$ on the $\mathrm{x}-\mathrm{y}$ plane by a motorized scanning stage (PLS-85, PI miCos $\mathrm{GmbH}$ ) to obtain a single-view 3D image (e.g., the left view). The sample is then re-scanned with the same configuration but with the right view laser beam to obtain an orthogonal-view 3D image.

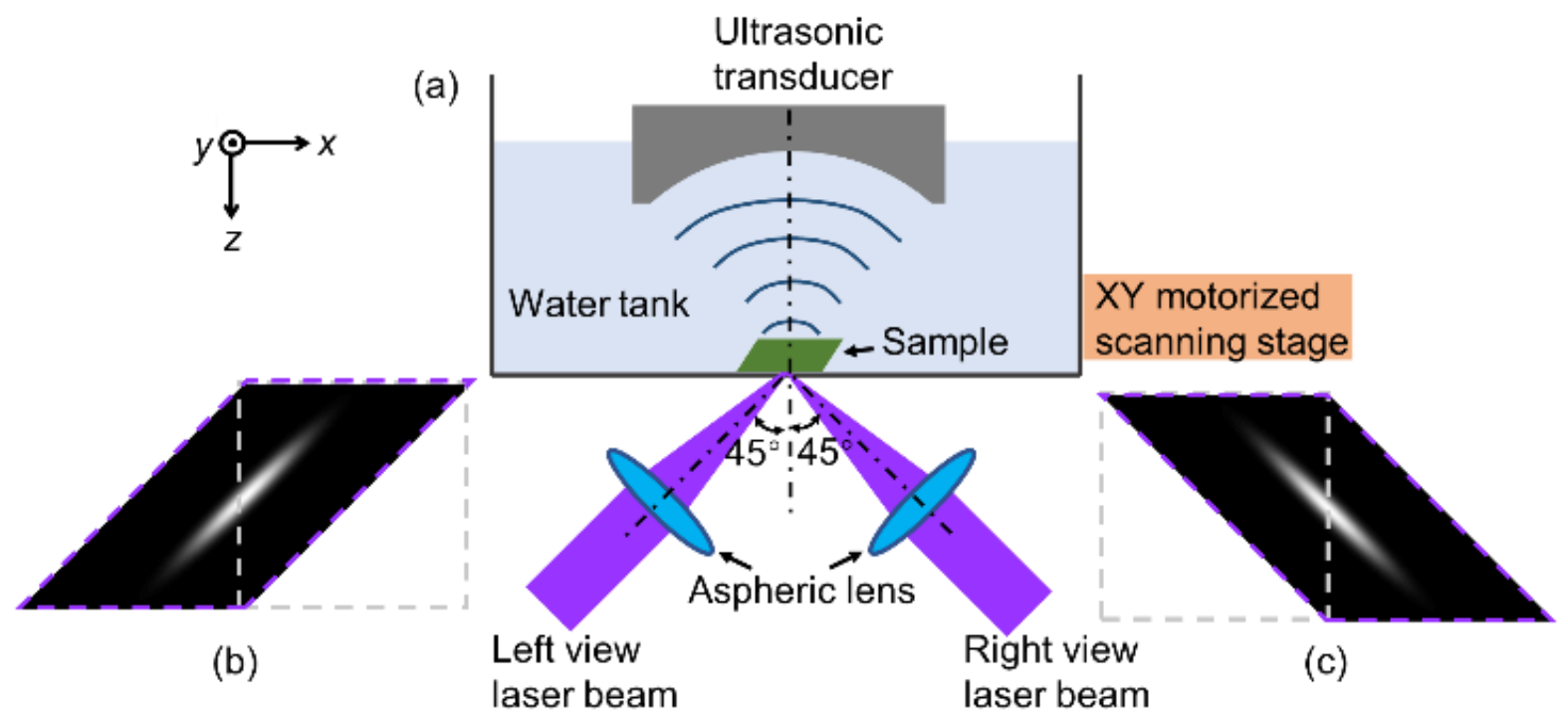

Figure 1. Schematic of the DV-OR-PAM system for improving resolution isotropy.

\section{RESULTS}

\section{Label-free DV-OR-PAM of sub-resolution beads}

Sub-resolution polystyrene beads with a $0.5 \mu \mathrm{m}$ average diameter, together with a target to be imaged, were embedded in agarose gel as registration markers. The beads were detected in the two view images and localized as point objects for registration. The sub-resolution beads were also used to extract the point spread functions (PSFs) in the two view images. The bead images were averaged and normalized to serve as the PSFs of the two view images. The PSFs were also affine transformed accordingly to co-register. The co-registered two view images and PSFs were then used for dual-view Richardson-Lucy deconvolution with 10 iterations to obtain a single dual-view image.

We used the sub-resolution beads to calibrate the resolutions of the original two images and the dual-view image. The volumetrically rendered 3D images of a single bead are shown in Figs. 2(a), 2(b), and 2(c) for the left view, the right view, and the dual view, respectively. Note that the original left and right view images were not affine transformed. The resolutions defined by the full width at half maximum are $3.0 \mu \mathrm{m}, 2.6 \mu \mathrm{m}$, and $1.8 \mu \mathrm{m}$ for the left view, the right view, and the dual view, respectively. The resolutions on the y-axis are $1.7 \mu \mathrm{m}, 1.7 \mu \mathrm{m}$ and $1.3 \mu \mathrm{m}$ for the left view, the right 
view, and the dual view, respectively. The acoustically determined axial resolution of the original left and right view images, far worse than the optically defined lateral resolutions, is $37 \mu \mathrm{m}$, which account for the elongated bead profiles and resolution anisotropy (Figs. 2(a), 2(b)). Dual-view imaging greatly improved the axial resolution to $1.8 \mu \mathrm{m}$ (i.e., by a factor of 20), making the bead profile nearly isotropic in 3D. In addition, the $\mathrm{x}$ and $\mathrm{y}$ resolutions are improved in the dual-view images due to deconvolution in the reconstruction algorithm.

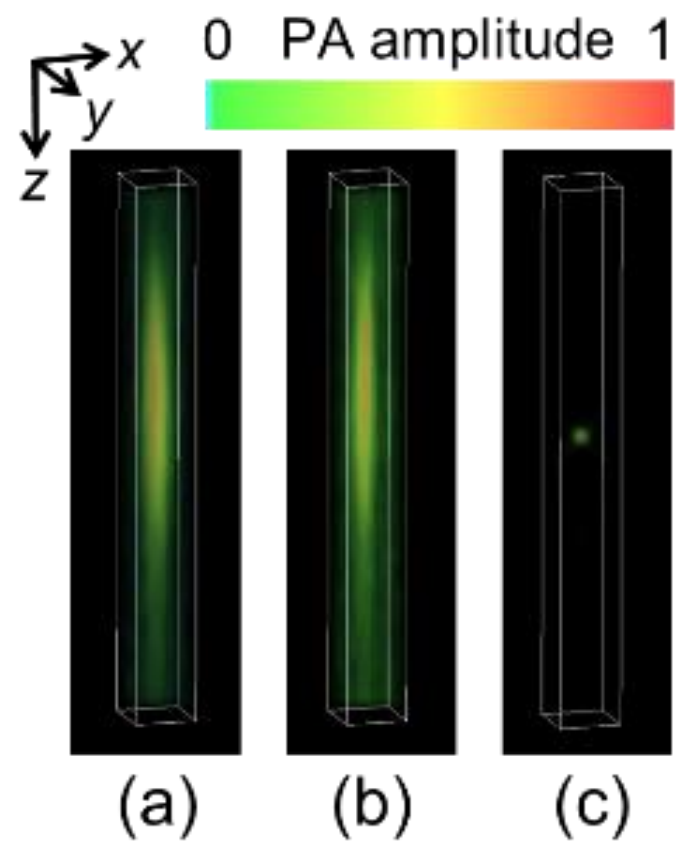

Figure 2. Resolution calibration for DV-OR-PAM using $0.5-\mu \mathrm{m}$-diameter beads as point objects. Volumetrically rendered bead images for (a) the left view, (b) the right view, and (c) the dual view, respectively.

\section{Label-free DV-OR-PAM of mouse brain slices}

We imaged mouse brain slices of $200 \mu \mathrm{m}$ thickness to further validate DV-OR-PAM. To enlarge the imaging area, the step size was set as $0.625 \mu \mathrm{m}$ with an imaging field of view over $1 \mathrm{~mm} \times 0.5 \mathrm{~mm}$ along the $\mathrm{x}$ and $\mathrm{y}$ axes. The absorption contrast at the $266 \mathrm{~nm}$ wavelength in mouse brains is predominantly cell nuclei [7], which appear to be the brightest features in the images. The $x-y$ maximum amplitude projection (MAP) images of the mouse brain slice are shown in Figs. 3(a), 3(b), and 3(c) for the left view, the right view, and the dual view, respectively. To better visualize the improved image quality of DV-OR-PAM, zoomed-in images of the red dashed boxes are shown in Figs. 3(d), 3(e), and 3(f). The cell nuclei can be better identified in the dual-view image with improvement in both resolution and signal-tonoise ratio (SNR) (by $\sim 10 \mathrm{~dB}$ ). Specifically, the cell nuclear profile in the blue dashed boxes, which cannot be resolved in the original two images (Figs. 3(g) and 3(h)), can be clearly revealed in the dual-view image as shown in Fig. 3(i). The detailed internal structure of the cell nuclei provided by DV-OR-PAM can potentially facilitate accurate cancer diagnosis $[19,20]$.

We have digitally extracted the image stacks on the $x-y$ plane of the red dashed regions at five different depths with an interval of $3.125 \mu \mathrm{m}$. In the original images, the image stacks at five different depths show a similar brightness because of the poor axial resolution, while in the dual-view image, different layers of cell nuclei are clearly demonstrated.

With the improved axial resolution, it is possible to reveal the accurate 3D shape of a cell nucleus as shown in Figs. 4(a)-(c), corresponding to the red dashed regions shown in Figs. 3(a)-(c). We quantitatively analyzed the cell nuclear volume, with a histogram plotted in Fig. 4(d). The average cell nuclear volumes are $5312 \pm 2202 \mu \mathrm{m}^{3}$ and $6665 \pm 2253$ $\mu \mathrm{m}^{3}$ for the left and right views, respectively. While in the dual-view image, the average cell nuclear volume is $778 \pm$ $396 \mu^{3}$, which is much closer to the realistic cell nuclear volume if an average diameter of $6 \mu \mathrm{m}$ and a spherical shape are assumed. Cell nuclear volumes and shapes are important diagnostic features for cancer diagnosis [8]. 

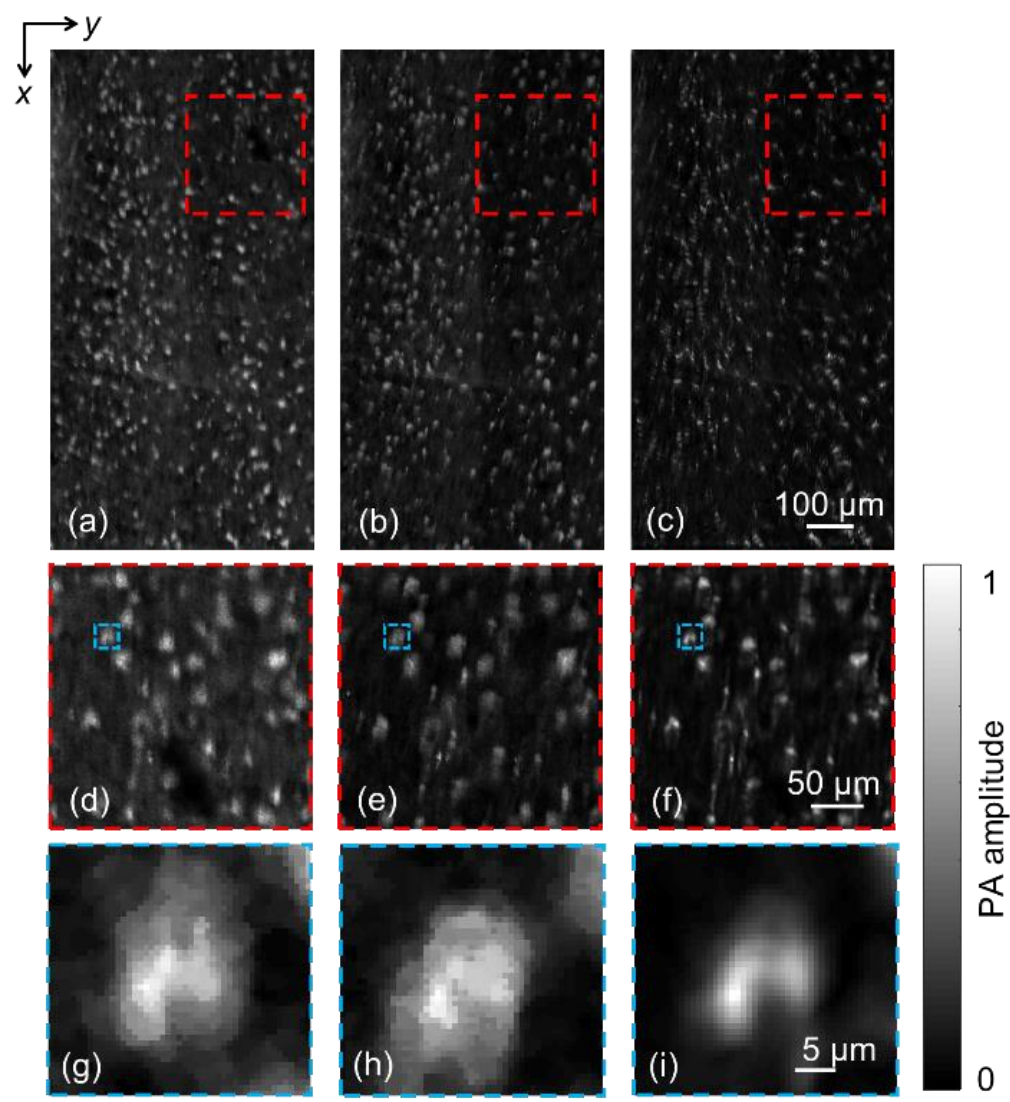

Figure 3. Imaging of a mouse brain slice. $x-y$ MAP images for (a) the left view, (b) the right view, and (c) the dual view, respectively. (d)-(f) Zoomed-in red dashed regions of (a)-(c). (g)-(i) Zoomed-in blue dashed regions of (d)-(f), showing the cell nuclear profile.
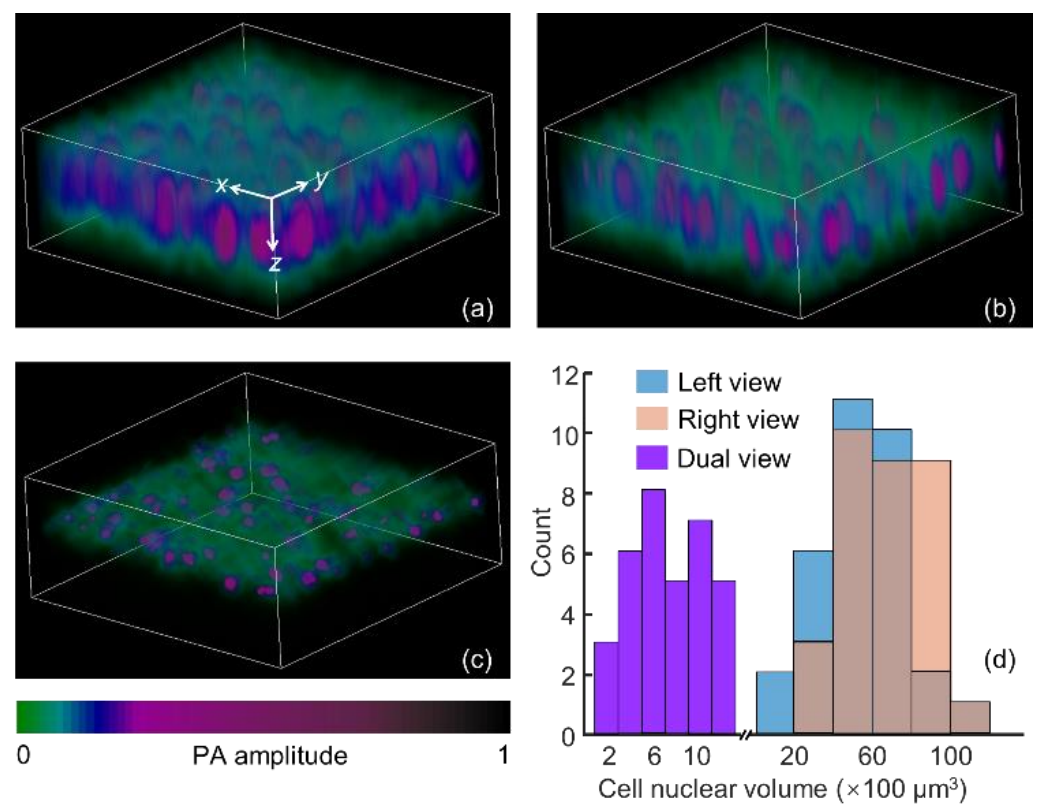

Figure 4. Volumetric rendering mouse brain slice images corresponding to the red dash regions in Fig. 3 for (a) the left view, (b) the right view, and (c) the dual view, respectively. Histogram of the cell nuclear volumes (d) imaged by the original two views and the dual view. 


\section{CONCLUSIONS}

In conclusion, a dual-view optical illumination for axial resolution improvement in OR-PAM was developed. Subresolution beads were used to validate the axial resolution improvement and resolution isotropy. The SNR of the dualview images was also improved. We used mouse brain slices to further demonstrate the efficacy of DV-OR-PAM for

quantitative cell nuclear analysis. The DV-OR-PAM is a promising tool for cancer diagnosis by providing quantitative $3 \mathrm{D}$ cell nuclear information.

\section{REFERENCES}

[1] Wang, L. V., and Yao J., "A practical guide to photoacoustic tomography in the life sciences," Nat. Methods 13, 627-638 (2016).

[2] Wong, T. T. W. et al. "Fast Label-free multilayered histology-like imaging of human breast cancer by photoacoustic microscopy," Sci. Adv. 3, e1602168 (2017).

[3] Wong, T. T. W. et al. "Label-free automated three-dimensional imaging of whole organs by microtomy-assisted photoacoustic microscopy," Nat. Commun. 8, 1386 (2017).

[4] Strohm, E. M. et al. "High frequency label-free photoacoustic microscopy of single cells," Photoacoustics 1, 49-53 (2013).

[5] Xie, Z. et al. "Pure optical photoacoustic microscopy," Opt. Express 19, 9027-9034 (2011).

[6] Liu, X. et al. "Label-free cell nuclear imaging by Grüneisen relaxation photoacoustic microscopy," Opt. Lett. 43, 947-950 (2018).

[7] Wang, L. et al. "Grueneisen relaxation photoacoustic microscopy," Phys. Rev. Lett. 113, 174301 (2014).

[8] Zink, D. et al. "Nuclear structure in cancer cells," Nat. Rev. Cancer 4, 677 (2004). 